\title{
Non-specific effects of measles, mumps, and rubella (MMR) vaccination in high income setting: population based cohort study in the Netherlands
}

${ }^{1}$ Centre for Infectious Disease
Control, National Institute
for Public Health and the
Environment, 3720 BA
Bilthoven, Netherlands
${ }^{2}$ Department of Paediatrics,
University Medical Centre
Utrecht, Utrecht, Netherlands
${ }^{3}$ Julius Centre for Health
Sciences and Primary Care,
University Medical Centre,
Utrecht, Netherlands
Correspondence to: M J Knol
mirjam.knol@rivm.nl
Additional material is published
online only. To view please visit
the journal online.
Cite this as: BMJ 2017;358:j3862
http://dx.doi.org/10.1136/bmj.j3862
Accepted: 8 August 2017

Accepted: 8 August 2017

\author{
Susanne M A J Tielemans, ${ }^{1}$ Hester E de Melker, ${ }^{1}$ Susan I M Hahné, ${ }^{1}$ Anna G C Boef, ${ }^{1}$ \\ Fiona R M van der Klis, ${ }^{1}$ Elisabeth A M Sanders, ${ }^{1,2}$ Marianne A B van der Sande, ${ }^{1,3}$ \\ Mirjam J Knol ${ }^{1}$
}

\section{ABSTRACT}

\section{OBJECTIVES}

To investigate whether measles, mumps, and rubella (MMR) vaccine has positive non-specific effects in a high income setting and to compare rates of hospital admissions for infections between children aged $\leq 2$ years who received live MMR vaccine and those who received an inactivated vaccine against diphtheria, tetanus, pertussis, polio, and Haemophilus influenzae type b (DTaP-IPV-Hib) as their most recent vaccination.

\section{DESIGN}

Nationwide population based cohort study.

\section{SETTING}

In the Netherlands, DTaP-IPV-Hib+pneumococcal vaccination (PCV) is recommended at ages $2,3,4$, and 11 months and MMR + meningococcal C (MenC) vaccination at age 14 months. Data from the national vaccine register were linked to hospital admission data.

\section{PARTICIPANTS}

1096594 children born in 2005-11 who received the first four DTaP-IPV-Hib+PCV vaccines.

\section{MAIN OUTCOME MEASURES}

Hazard ratio for admission to hospital for infection in children with MMR+MenC compared with the fourth DTaP-IPV-Hib+PCV as their most recent vaccination. Cox regression was performed with most recent vaccination as time dependent variable, adjusted for potential confounders. Analyses were repeated with admission for injuries or poisoning as a negative control outcome. In addition, rate of admission for infection was compared between the fourth and third DTaP-IPV-Hib+PCVas most recent vaccination.

\section{WHAT IS ALREADY KNOWN ON THIS TOPIC}

Live attenuated vaccines (such as measles) could have beneficial non-specific effects and inactivated vaccines (such as DTP) could have deleterious non-specific effects

The evidence for non-specific effects of vaccines remains weak as most evidence comes from observational studies that are vulnerable to bias

\section{WHAT THIS STUDY ADDS}

Healthy vaccinee bias at least partly explains the observed lower rate of hospital admission for infection after MMR vaccination; this lower rate is associated with receipt of any additional vaccine and not specifically with MMR

The presence of non-specific effects of MMR vaccination cannot be excluded, but the possible non-specific effects cannot be distinguished from bias

The findings emphasise the importance of caution in the interpretation of findings from observational studies on non-specific effects of vaccination

\section{RESULTS}

Having had MMR+MenC as the most recent vaccination was associated with a hazard ratio of 0.62 ( $95 \%$ confidence interval 0.57 to 0.67 ) for admission to hospital for infection and 0.84 ( 0.73 to 0.96$)$ for injuries or poisoning, compared with the fourth DTaP. IPV-Hib+PCV as most recent vaccination. The fourth DTaP-IPV-Hib+PCV as most recent vaccination was associated with a hazard ratio of 0.69 (0.63 to 0.76 ) for admission to hospital for infection, compared with the third DTaP-IPV-Hib+PCV as most recent vaccination.

\section{CONCLUSIONS}

Healthy vaccinee bias could at least partly explain the observed lower rate of admission to hospital with infection after MMR vaccination. The lower rate is associated with receipt of any additional vaccine, not specifically MMR vaccine. This emphasises the caution required in the interpretation of findings from observational studies on non-specific effects of vaccination.

\section{Introduction}

Vaccines against measles; diphtheria, pertussis, and tetanus (DTP); and polio have led to large declines in morbidity and mortality from the targeted diseases. ${ }^{12}$ It has been suggested that these vaccines could also affect morbidity and mortality from infections other than those targeted by the vaccines-that is, that they have non-specific effects. ${ }^{3}$ Several studies observed beneficial non-specific effects of live attenuated vaccines (such as measles and BCG) and deleterious non-specific effects of inactivated vaccines (such as DTP vaccine), with, in general, stronger effects in girls than in boys. ${ }^{45678910}$ Moreover, the sequence of vaccination could be important. Receipt of a live attenuated measles vaccine after an inactivated DTP vaccine might be associated with lower morbidity and mortality, compared with receipt of a DTP vaccine after or at the same time as a measles vaccine. ${ }^{111213141516}$ Which immunological mechanisms could underlie these potential non-specific effects of vaccination on susceptibility to infectious disease is currently unknown. Trained innate immunity, which depends on epigenetic reprogramming of innate immune cells, could explain some of the non-specific effects. ${ }^{1718}$ Another potential mechanism is through $\mathrm{T}$ cell mediated cross reactivity. ${ }^{18}$

Few studies on non-specific effects have been performed in high income countries, which have low rates of infant mortality from infectious diseases. 
The public health relevance of non-specific effects of vaccines in high income countries is largely unknown. Two nationwide Danish studies reported measles, mumps, and rubella (MMR) vaccine to be associated with a $16 \%$ lower rate of hospital admissions for infectious disease $\mathrm{e}^{10}$ and a $22 \%$ lower rate of hospital contacts for respiratory syncytial virus ${ }^{19}$ compared with DTP-IPV-Hib as most recent vaccine. No differences were observed between boys and girls. The Danish studies used timing of MMR vaccination as the (time varying) exposure.

Most of the evidence on non-specific effects of vaccines originates from observational studies, which are prone to bias. ${ }^{20}$ An example is healthy vaccinee bias (also known as healthy user bias or frailty bias), which occurs when children who are more susceptible to illness are vaccinated later or not at all, resulting in an overestimation of the beneficial effect of the next vaccination. Recently, two systematic reviews of the potential non-specific effects of DTP and measles vaccines, which were commissioned by the World Health Organization strategic advisory group of experts (SAGE), were published. ${ }^{21}$ Findings suggest that receipt of measles vaccine reduces overall mortality more than would be expected through the effects on the targeted disease, while DTP vaccination might be associated with an increase in all cause mortality. ${ }^{21}$ As most evidence came from observational studies (10 observational studies for DTP ${ }^{11} 12^{22232425262728}$; four trials ${ }^{6293031}$ and 17 observational studies ${ }^{11} 1213222832333435363738$ 39404142 for measles), however, the conclusion was that these findings should be interpreted with caution.

We set out to reproduce the findings of the Danish studies on non-specific effects of MMR vaccination in a population based nationwide cohort study of more than a million Dutch children using similar methods. We investigated the rate of hospital admissions related to infectious disease after receipt of the live attenuated MMR vaccine (given at the same time as vaccination against meningococcal disease group $\mathrm{C}$, MenC) versus inactivated DTP containing vaccine (also includes vaccinations against polio (inactivated polio vaccine, IPV) and Haemophilus influenzae type $\mathrm{b}$ and given at the same time as a vaccination against pneumococcal disease) as most recent vaccine. In addition, we explored whether healthy vaccinee bias was present.

\section{Methods}

\section{Study design}

The Dutch national immunisation programme was implemented in 1957 and is coordinated and evaluated by the Dutch National Institute for Public Health and the Environment. Table 1 shows the recommended vaccines included in the programme in the first 24 months of life, and table A in appendix 1 shows the years of introduction into the programme. The DTaP-IPV-Hib vaccine, recommended at ages 2, 3, 4, and 11 months, consists of vaccinations against diphtheria, tetanus, pertussis (acellular), polio, and conjugate vaccine against $H$ influenzae type $\mathrm{b}$ (Hib) and is administered simultaneously with a multivalent
Table 1 | Vaccines recommended in first 24 months of life according to Dutch national immunisation programme Age (months) Vaccinations

\begin{tabular}{|c|c|}
\hline 2 & DTaP-IPV-Hib(-HepB since August 2011)+PCV \\
\hline 3 & DTaP-IPV-Hib(-HepB since August 2011)+PCV \\
\hline 4 & DTaP-IPV-Hib(-HepB since August 2011)+PCV \\
\hline 11 & DTaP-IPV-Hib(-HepB since August 2011)+PCV \\
\hline 14 & MMR + MenC \\
\hline $\begin{array}{l}\text { DTa } \\
\text { B; } \\
\text { Me }\end{array}$ & $\begin{array}{l}\text { nus, pertussis (acellular); HepB=hepatitis } \\
\text { pe b; IPV=inactivated polio vaccine; } \\
\text { disease group C; MMR=measles, mumps, rubella; } \\
\text { onjugate vaccine. }\end{array}$ \\
\hline
\end{tabular}

conjugate vaccination against pneumococcal disease (PCV). The vaccination against MMR is recommended at age 14 months and administered simultaneously with vaccination against meningococcal disease serogroup C (MenC). Only the MMR vaccine is a live vaccine; the other vaccines are inactivated/non-live vaccines.

\section{Vaccination data}

In 2005, an electronic national immunisation register "Præventis" was implemented in the Netherlands. The register is linked to the population register. This means that all children and young people aged under 19 who are officially registered in the Netherlands are included in the immunisation register. Praeventis does not include undocumented children living in the Netherlands. Parents receive invitation letters automatically created by Præventis to get their child(ren) vaccinated at a specific date and time at a healthy children clinic near their homes according to the immunisation programme. Data on administered vaccinations (that is, vaccine characteristics, dose, date of administration) are entered in Præventis. ${ }^{43}$

\section{Hospital admission data}

The national medical register administeredby Dutch hospital data provided data on hospital admissions from 1 January 2005 to 31 December 2012. Dutch hospital data requests hospitals and university medical centres in the Netherlands to voluntarily supply data on admissions. The coverage of all hospital admissions decreased with time, with 3\% missing in 2005 to $25 \%$ in $2012 .{ }^{44}$

Primary and secondary discharge diagnoses and dates of admissions were available from the register. Diagnoses were coded according to the ICD-9-CM (international classification of diseases, ninth revision, clinical modification). We included upper respiratory infections, lower respiratory infections, gastrointestinal infections, and other infections (table B in appendix $1)$. In the main analysis we included only hospital admissions that lasted more than one day (and thus included an overnight stay) to exclude day admissions related to planned examinations and surgeries. In a sensitivity analysis, we included all admissions.

\section{Covariates}

Præventis provided data on sex, parents' country of birth, and postcode, and Statistics Netherlands 
Children in study population ( $n=1356926$ )

Excluded $(n=260332)$

Did not receive four recommended DTaP-IPV-Hib vaccines ( $n=102422)$

Received 4th DTaP-IPV-Hib vaccine before age 9 months $(n=322)$

Received 4th DTaP-IPV-Hib vaccine before age 20 months $(n=5235)$

Received 4th DTaP-IPV-Hib vaccine afte

$\rightarrow \quad 31$ December $2012(n=2586)$

Received MMR vaccine before age 12 months, before 4th DTaP-IPV-Hib vaccine, or simultaneously with 4th DTaP-IPV-Hib vaccine $(n=67408)$

Had birth weight $<500 \mathrm{~g}(\mathrm{n}=31)$

Had missing data on covariates (birth weight, gestational age, maternal age, parental country of birth, and postcode) $(n=81389)$

Missing data because of migration $(n=939)$

Children in final study population ( $n=1096594)$

Fig 1 | Flow chart of children included in study population according to compliance with vaccination schedule

provided data on death, migration, and parental educational level. Parental educational level was classified as low (elementary education or prevocational education), medium (senior general secondary education, pre-university education, or vocational education), or high (college or university). The Netherlands perinatal registry provided data on birth weight, gestational age, maternal age, and parity (as a proxy for number of siblings).

\section{Linkage of data sources}

The population register (the municipal personal records database) was the main database to which all databases were linked to get a unique anonymised number with which the different databases could be linked. Of all 1357461 children that were included in Præventis, 1356926 (>99\%) were successfully linked with the population register with a unique personal identifier, the citizen service number. Data from the national medical register, Statistics Netherlands, and the Netherlands perinatal registry were linked to the population register by using probability matching, based on sex, date of birth, and postcode. For the national medical register, unique records were successfully linked, ranging from $97.3 \%$ in 2005 to $98.7 \%$ in $2012 .^{45}$

\section{Population for analysis}

For the present study, vaccination data from 1356926 Dutch children born from 1 January 2005 to 31 December 2011 who were all eligible for the routine immunisation programme were available from the electronic national immunisation register Præventis (fig 1). Of these children, 93\% received the first four recommended DTaP-IPV-Hib+PCV vaccines and were eligible for inclusion in this study. We excluded children who did not receive these DTP containing vaccinations $(n=102422)$ to limit the possibility of bias attributable to factors related to low vaccination coverage (such as refusal based on religion). We also excluded children who received the fourth DTaPIPVHib+PCV vaccine either before age 9 months $(n=322)$, after age 20 months $(n=5235)$, after 31 December $2012(\mathrm{n}=2586)$, after the MMR vaccine ( $\mathrm{n}=18110)$, or simultaneously with the MMR vaccine ( $\mathrm{n}=4333$ ). In addition, we excluded children who received an MMR vaccine before age 12 months $(n=44965)$ because these children are recommended to receive another MMR vaccine at 14 months and therefore follow a different vaccination schedule than the other children in the study population. After exclusion of children with a birth weight $<500$ $\mathrm{g}(\mathrm{n}=31)$, missing data on covariates (birth weight, gestational age, maternal age, parental country of birth, and postal code) ( $\mathrm{n}=81389)$, or missing data because of migration ( $n=939), 1096594$ children (81\%) remained for analysis.

\section{Statistical analysis}

Main analysis

Cox proportional hazards models were used to estimate hazard ratios for hospital admissions related to infectious disease according to the most recent vaccination (MMR+MenC versus fourth DTaP-IPV$\mathrm{Hib}+\mathrm{PCV}$ ), with last received vaccination included as a time varying variable changing at the age of receipt of the MMR+MenC vaccine (statistical code provided in appendix 2). Children entered the model at the age of receipt of the fourth DTaP-IPV-Hib+PCV and were followed until the age of hospital admission for infection (in case of an event) or were censored at death, age 24 months, age of emigration, or age on 31 December 2012, whichever came first. Age was used as timescale for the Cox regression. We included age at MMR+MenC vaccination as a time variable exposure and used age as a timescale in the Cox regression; this effectively means that at each age (in days) we compared children who had already received the MMR+MenC vaccine with children who had not yet received the $M M R+M e n C$ vaccine, thereby adjusting for age.

Analyses were stratified by date of birth to fully control for age, season, and calendar year. Associations were adjusted for sex, chronic diseases $(\mathrm{Y} / \mathrm{N})$ before baseline (age 9 months; list of ICD-9 codes, shown in table $\mathrm{C}$ in appendix 1 ), admission to hospital for any reason in the month before baseline (the fourth DTaP-IPV-Hib+PCV is received from age 9 months onwards, a fixed month of 8 months of age was chosen for this variable) $(\mathrm{Y} / \mathrm{N})$, birth weight, gestational age, maternal age and parity $(1,2,3$, and 24), parents' country of birth (two Dutch parents, one Dutch and one non-Dutch parent, and two non-Dutch parents), and postcode in three digits. Analyses were stratified by type of infection-for instance, upper respiratory, lower respiratory, gastrointestinal, and other infections. Sex and birth year were examined as potential effect modifiers by an interaction test and stratified analysis. Because a lot of data were missing for level of parental educational, we additionally adjusted associations for level (low, medium, or 
high) in a subsample of $79 \%$ of eligible children with complete information.

The same analysis was performed with admission for injury or poisoning (ICD-9-CM codes 800-999) as a negative control outcome. ${ }^{46}$

Analyses were performed with SAS version 9.2 (SAS Institute, Cary, NC) and Stata 14 (StataCorp, College Station, TX).

\section{Sensitivity analyses}

Firstly, we performed the same analysis as described above with all hospital admissions for infections taken into account, thus also including day admissions without an overnight stay.

Secondly, we performed another analysis taking into account repeated hospital admissions with the Andersen-Gill model ${ }^{47}$ as an extension of the Cox model. We excluded all hospital admissions for infection that occurred less than 14 days after a previous discharge because multiple admissions within a short period could be attributable to the same infection. In cases of a previous hospital admission, children re-entered the study 14 days after discharge and were followed until a next admission or censored at death, age 24 months, or age at 31 December 2012, whichever came first.

In a third analysis, we compared the fourth with the third DTaP-IPV-Hib+PCV vaccination. Vaccination was included as a time varying variable changing at the age of the fourth vaccination. Children entered the model from the age of receipt of the third vaccination and were followed until the age of admission for infection or were censored at death, age of MMR+MenC vaccination, age 14 months, or age on 31 December 2012, whichever came first.

\begin{tabular}{|c|c|}
\hline & Data \\
\hline Median (IQR) age at fourth DTaP-IPV-Hib vaccination (months) & $11.2(11.0-11.6)$ \\
\hline Median (IQR) age at MMR vaccination (months)* & $14.3(14.0-14.8)$ \\
\hline Boys & $561407(51.2)$ \\
\hline Median (IQR) birth weight (g) & $3460(3100-3810)$ \\
\hline Median (IQR) gestational age (weeks) & $39.9(38.7-40.7)$ \\
\hline Median (IQR) maternal age at birth of child (years) & $31(27-34)$ \\
\hline \multicolumn{2}{|l|}{ Maternal parityt: } \\
\hline One & $505851(46.1)$ \\
\hline Two & $400703(36.5)$ \\
\hline Three & $138565(12.6)$ \\
\hline Four or more & $51473(4.7)$ \\
\hline \multicolumn{2}{|l|}{ Highest parental educational levelł: } \\
\hline Low & $115205(13.2)$ \\
\hline Medium & $310445(35.6)$ \\
\hline High & $445544(51.1)$ \\
\hline \multicolumn{2}{|l|}{ Parental country of birth: } \\
\hline Netherlands & $834580(76.1)$ \\
\hline Netherlands and foreign & $142555(13.0)$ \\
\hline Foreign & $119457(10.9)$ \\
\hline Chronic disease of child & $27430(2.5)$ \\
\hline Hospital admission for any reason at age 8 months & $11706(1.1)$ \\
\hline \multicolumn{2}{|c|}{$\begin{array}{l}\text { IQR=interquartile range. } \\
\text { * Data available for } 1092625 \text { children. } \\
\text { † Represents number of childbirths of mother. } \\
\text { ‡ Highest educational level of household (either parent). Data available for } 871194 \text { children. }\end{array}$} \\
\hline
\end{tabular}

\section{Patient involvement}

No patients were involved in setting the research question or the outcome measures, nor were they involved in developing plans for recruitment, design, or implementation of the study. No patients were asked to advise on interpretation or writing up of results. There are no plans to disseminate the results of the research to study participants or the relevant patient community.

\section{Results}

\section{Study population}

Table 2 shows characteristics of the 1096594 children included in the analyses. Children received the fourth DTaP-IPV-Hib+PCV vaccination at a median age of 11.2 months and the MMR+MenC vaccination at a median age of 14.3 months. Almost all children received the MMR vaccination (99.6\%) (fig 2), and about $99 \%$ of all children received PCV with the DTaP-IPV-Hib vaccines and the MenC vaccine with the MMR vaccine. Most children had at least one Dutch parent (89.1\%), and about half of the children had at least one parent with higher education $(51.1 \%$ of all children with data on parental educational level). About $1 \%$ of all children were admitted to hospital for any reason at age 8 months, and $2.5 \%$ had previously had a diagnosis of a chronic disease before receipt of the fourth DTaP-IPV$\mathrm{Hib}+\mathrm{PCV}$ vaccine.

\section{MMR+MenC $v$ fourth DTaP-IPV-Hib+PCV in relation to admission for infection (main analysis)}

During 1061242 person years of follow-up, 10961 children were admitted to hospital for more than one day (this was $26 \%$ of all admissions for infection, meaning that $74 \%$ of all admissions for infection were day admissions) for an infection (admission rate $10 / 1000$ person years). Admission rates declined with age, from $15 / 1000$ person years at age 12 months to $7 / 1000$ person years at age 24 months (fig 3 ). Similarly, admission rates declined with age in those who received MMR+MenC as their most recent vaccination (average admission rate of $9 / 1000$ person years). In children whose most recent vaccination was the fourth DTaP-IPV-Hib+PCV, admission rates slightly declined

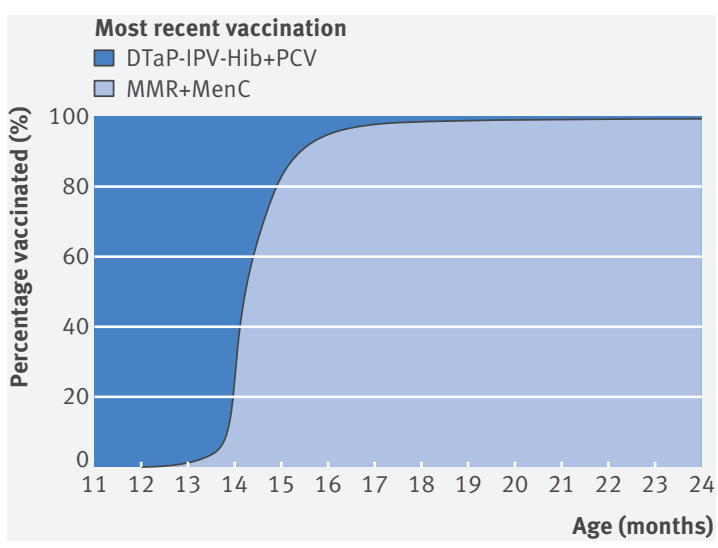

Fig 2 | Proportion of children according to most recent vaccination with DTaP-IPV-Hib+PCV and MMR+MenC, based on 1096594 children included in present study 


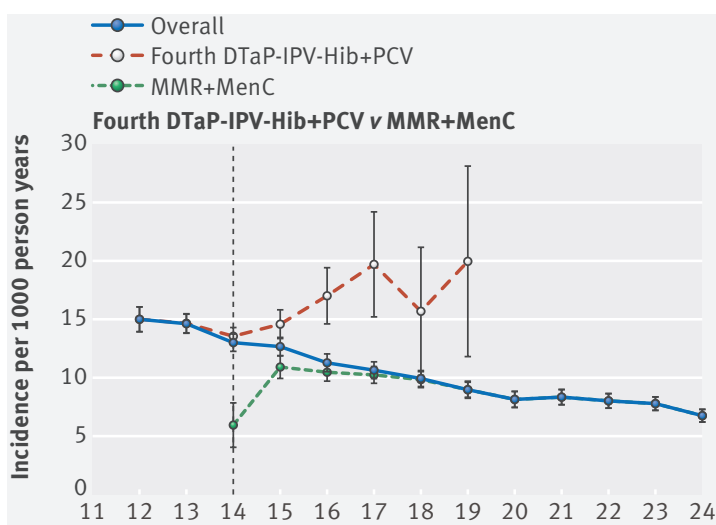

Age (months)

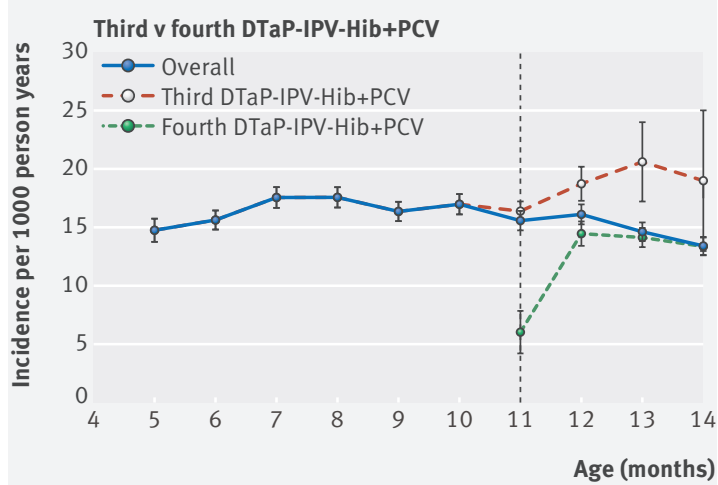

Fig 3 | Hospital admissions rates for infection with 95\% confidence intervals for fourth DTaP-IPV-Hib+PCV $v$ $M M R+M e n C$ as most recently received vaccination (in top panel line for fourth DTaP-IPV-Hib+PCV represents admission rate in children who had not yet received $M M R+M e n C$ vaccination) and for third $v$ fourth DTaP-IPV$\mathrm{Hib}+\mathrm{PCV}$ (bottom panel). Dashed vertical lines represent recommended age of vaccination (top: $M M R+M e n C$ at age 14 months; bottom: fourth DTaPIPVHib+PCV at age 11 months). Each estimate represents incidence of month before that month-for example, estimate at age 12 months comprises incidence for age 335-364 days. Note that incidence of admissions for MMR vaccinated individuals aged 12-14 months was not reported because too few children in study population received MMR at age 12-14 months (see fig 2), and, of these, $<10$ were admitted to hospital for infection. Because of privacy reasons, we cannot report data that apply to $<10$ individuals until 14 months, which is the median age of receipt of MMR+MenC, and increased thereafter until age 17 months. Admission rates at age 17 months were 1.8 times higher in those whose most recent vaccination was the fourth DTaPIPVHib+PCV, compared with the overall admission rates (20/1000 $v 11 / 1000$ person years). The average admission rate in those whose most recent vaccination was the fourth DTaP-IPV-Hib+PCV was $14 / 1000$ person years.

Compared with the fourth DTaP-IPV-Hib+PCV as most recent vaccination, receipt of $\mathrm{MMR}+\mathrm{MenC}$ as the most recent vaccination was associated with a age adjusted hazard ratio of 0.60 (95\% confidence interval 0.55 to 0.65 ) for hospital admissions related to infectious disease (table 3). After additional adjustment for sex, chronic disease, admissions at age 8 months, birth weight, gestational age, maternal age and parity, parents' country of birth, and postcode, the hazard ratio was 0.62 (0.57 to 0.67$)$. No effect modification was observed for sex $(\mathrm{P}=0.55$ for interaction $)$ and birth cohort ( $\mathrm{P}=0.29$ for interaction) (table $\mathrm{D}$ in appendix 1 ). Additional adjustment for level of parents' educational in the subsample of children with available data changed the results marginally (0.64, 0.58 to 0.70 ). When we took repeated hospital admissions for infections into account (event rate 11/1000 person years), the adjusted hazard ratio $(0.72,0.66$ to 0.79$)$ was slightly increased.

\section{Type of infection}

Of all 10961 hospital admissions related to infection, $43 \%$ were for gastrointestinal infections, $40 \%$ for upper respiratory infections, $31 \%$ for lower respiratory infections, and $17 \%$ for other infections. The hazard ratio for admission according to most recent vaccination ranged between 0.54 (95\% confidence interval 0.48 to 0.62 ) for upper respiratory infections to 0.70 (95\% 0.61 to 0.80$)$ for gastrointestinal infections (table $\mathrm{E}$ in appendix 1).

\section{All admissions (including day admissions)}

When we took into account all hospital admissions for infections, including day admissions without an overnight stay, 41976 children were admitted to hospital for infection during 1047465 person

\begin{tabular}{|c|c|c|c|c|}
\hline \multirow{2}{*}{ Outcome } & & & $\mathrm{HR}(95 \% \mathrm{Cl})^{*}$ & \\
\hline & Most recent vaccination & Events/person years & Age adjusted & Fully adjusted $t$ \\
\hline \multirow[t]{2}{*}{ Infections } & Fourth DTaP-IPV-Hib+PCV & $4111 / 284786$ & 1.00 & 1.00 \\
\hline & MMR+MenC & $6850 / 776456$ & $0.60(0.55$ to 0.65$)$ & $0.62(0.57$ to 0.67$)$ \\
\hline \multirow[t]{2}{*}{ Injuries or poisoning } & Fourth DTaP-IPV-Hib+PCV & $3605 / 285676$ & 1.00 & 1.00 \\
\hline & MMR+MenC & $5150 / 782738$ & 0.81 (0.71 to 0.93$)$ & 0.84 (0.73 to 0.96$)$ \\
\hline \multirow[t]{2}{*}{ Infections } & Third DTaP-IPV-Hib+PCV & $10654 / 639484$ & 1.00 & 1.00 \\
\hline & Fourth DTaP-IPV-Hib+PCV & $3185 / 231001$ & $0.66(0.60$ to 0.72$)$ & 0.69 (0.63 to 0.76$) \neq$ \\
\hline
\end{tabular}


years of follow-up. Of these admissions, $83 \%$ were for upper respiratory infections, $9 \%$ for lower respiratory infections, $12 \%$ for gastrointestinal infections, and $6 \%$ for other infections. Receipt of MMR+MenC as the most recent vaccination instead of the fourth DTaP-IPV-Hib+PCV was associated with a fully adjusted hazard ratio of 0.40 (95\% confidence interval 0.38 to 0.41 ) for hospital admissions related to infectious disease, which was driven mainly by upper respiratory infections (table $\mathrm{F}$ in appendix 2).

\section{Negative control outcome}

During 1068414 person years of follow-up, 5150 children were admitted to hospital for more than one day because of injury or poisoning (negative control outcome). After full adjustment, we observed the hazard ratio was 0.84 (95\% confidence interval 0.73 to 0.96 ) for admission for injuries or poisoning with $\mathrm{MMR}+\mathrm{MenC}$ as the most recent vaccination compared with the fourth DTaP-IPV-Hib+PCV as most recent vaccination (table 3 ). When we also considered admissions without an overnight stay, the fully adjusted hazard ratio was 0.80 (0.71 to 0.89) (table F in appendix 1).

\section{Fourth $v$ third DTaP-IPV-Hib+PCV in relation to hospital admission for infection}

During 870485 person years of follow-up, 13839 children were admitted for more than one day for infection (admission rate 16/1000 person years). The average admission rate in those with the fourth DTaPIPV-Hib+PCV as their most recent vaccination was $14 / 1000$ person years. In those with the third DTaP-IPV$\mathrm{Hib}+\mathrm{PCV}$ as their most recent vaccination, admission rates were quite stable until age 11 months, which is the median age of receipt of the fourth vaccination, and increased thereafter until age 14 months (fig 3). Admission rates at age 14 months were 1.5 times higher in those with the third DTaP-IPV-Hib+PCV as their most recent vaccination compared with the overall admission rates (19/1000 v 13/1000 person years). The average admission rate in those with third vaccination as their most recent was $17 / 1000$ person years.

Receipt of the fourth DTaP-IPV-Hib+PCV as the most recent vaccination was associated with a age adjusted hazard ratio of 0.66 (95\% confidence interval 0.60 to 0.72 ) for hospital admissions for infectious disease compared with the third DTaP-IPV-Hib+PCV vaccination as the most recent (table 3). After additional adjustment for sex, chronic disease, birth weight, gestational age, maternal age, parity, parents' country of birth, and postcode, the hazard ratio was 0.69 (0.63 to 0.76). When we took into account admissions without an overnight stay, the fully adjusted hazard ratio was 0.48 (0.46 to 0.51 ) (table $\mathrm{F}$ in appendix 1$)$. The adjusted hazard ratios by type of infection ranged from 0.59 ( 0.47 to 0.74 ) for other infections to 0.79 (0.67 to 0.92) for lower respiratory infections (table $\mathrm{E}$ in appendix 1).

\section{Discussion}

In more than a million Dutch children aged 1124 months, there was a 38\% lower rate of hospital admissions related to infectious disease in those who had MMR+MenC as their most recent vaccination, compared with those who had DTaP-IPV-Hib+PCV as their most recent vaccination. We also observed a $16 \%$ lower rate of admission for injuries or poisoning (negative control outcome) in children with MMR+MenC as their most recent vaccination. Moreover, there was a 31\% lower rate of admissions related to infectious disease for children with the fourth DTaP-IPV-Hib+PCV as their most recent vaccination, compared with the third as their most recent vaccine. These findings suggest that a lower rate of admission is associated with adherence to the routinely recommended schedule. It is likely that healthy vaccinee bias was present and (at least partly) explains the lower rate of infections that we observed for receipt of an additional vaccine, rather than being an effect of specifically receiving MMR. The findings of this large scale observational study on non-specific effects emphasise the extreme difficulty in interpreting such results given the likely presence of healthy vaccinee bias.

We observed that after the median age of receipt of the next vaccine, which was MMR+MenC at 14 months in the MMR+MenC versus DTaP-IPV-Hib+PCV analysis and the fourth DTaP-IPV-Hib+PCV at 11 months in the analysis of fourth versus third DTaP-IPV-Hib+PCV, admission rates among those who deviated from the recommended schedule suddenly increased compared with the overall admissions rates. This suggests that vaccination is postponed in children who are more prone to admission. The confounders that we included in the analysis did not considerably change the hazard ratio, so apparently these confounders do not explain the increased risk of admission in children in whom vaccination is postponed. An unmeasured confounder could be acute illness, which might be associated with timing of vaccination and rate of admission. The lower rate of admission for infection for the fourth DTaP-IPV$\mathrm{Hib}+\mathrm{PCV}$ as most recent vaccination compared with the third also suggests that receipt of an additional vaccination (and therefore adherence to the routinely recommended schedule) is followed by a lower rate of admission, and this is thus not a finding that can be attributed to MMR specifically. This raises concerns for past and future observational studies on non-specific effects and emphasises that evidence from randomised trials that also investigate different sequences of vaccines is needed to draw conclusions on this matter, as was concluded by the recently published WHOSAGE review. ${ }^{21}$

In a nationwide population based cohort of about 500000 Danish children, ${ }^{10}$ the rate of hospital admissions related to infectious disease was $14 \%$ lower in those with MMR as their most recent vaccination compared with DTaP-IPV-Hib as their most recent vaccination. Their report of non-specific effects of MMR vaccination was strengthened by the fact that they found a $62 \%$ higher rate of hospital admissions 
related to infectious disease in children who received DTaP-IPV-Hib after MMR vaccination, and they did not find any effect of MMR vaccination on emergency department visits after unintentional injury. A difference between the Danish study and our study is that there was more variation in the Danish studyin the age at MMR vaccination (median 15.8 (interquartile range15.2-17.0) v 14.3 (14.0-14.8)), which could be attributed to a different vaccination system. In the Netherlands, appointments for each vaccination are made in child health clinics in advance. In Denmark, parents have to make an appointment with the GP themselves for their children to receive vaccinations. This might have led to more random variation in the age at MMR vaccination and therefore proportionally less variation from factors related to the child's health in Denmark than in the Netherlands. The system in the Netherlands, with a more fixed schedule as result of prescheduled appointments, more clearly shows the existence of healthy vaccinee bias. This could explain the discrepancy between findings. It seems likely, however, that healthy vaccinee bias is also present in the Danish study. We therefore consider it likely that the lower rate of infection as estimated in the Danish setting overestimates any non-specific effects.

Several randomised controlled trials have been performed in low income countries to assess nonspecific effects of vaccines containing measles on all cause mortality. The pooled relative risk from four randomised controlled trials was 0.74 (95\% confidence interval 0.51 to 1.07), pointing towards beneficial effects of receipt of such vaccines. ${ }^{21}$ Like these vaccines, BCG vaccination, another live attenuated vaccine, has also been associated with non-specific effects in low income countries. ${ }^{21} \mathrm{~A}$ recently published randomised controlled study on BCG vaccination at birth in Denmark, however, did not find an effect on all cause hospital admissions ${ }^{48}$ or childhood infections reported by parents. ${ }^{49}$

Although we show that healthy vaccinee bias is probably present in our analysis, the impact of this bias is hard to quantify, and we cannot exclude that non-specific effects are still present in our study. If we assume that the lower rate of infection after the fourth versus the third DTaP-IPV-Hib+PCV vaccination is due to healthy vaccinee bias, the difference in hazard ratios between the MMR+MenC versus DTaP-IPV-Hib+PCV (hazard ratio of $0.62,95 \%$ confidence interval 0.57 to 0.67 ) and the fourth versus the third DTaP-IPV$\mathrm{Hib}+\mathrm{PCV}(0.69,0.63$ to 0.76$)$ could be ascribed to nonspecific effects. Furthermore, in the MMR+MenC versus DTaP-IPV-Hib+PCV analysis the effect was stronger for respiratory infections than for other infections, but this was not the case for the fourth versus the third DTaP-IPV-Hib+PCV analysis. As non-specific effects have been found to be stronger for respiratory infections, ${ }^{10} 19$ these findings could indicate the presence of non-specific effects of MMR. This is rather indirect evidence, comparing different populations of children, however, and therefore not robust. These findings emphasise the difficulty of investigating non- specific effects of vaccination in observational studies. This is also confirmed by our findings for the negative control outcome. We observed a 14\% (95\% confidence interval $4 \%$ to $27 \%$ ) lower rate of admission for injuries or poisoning, which can obviously not be explained by non-specific effects. This finding could also be explained by healthy vaccinee bias but to a lesser extent.

It should be noted that in the Netherlands, the DTaPIPV-Hib vaccine is administered with a multivalent conjugate vaccination against pneumococcal disease and the MMR vaccine is administered with vaccination against MenC. We cannot exclude the possibility that beneficial non-specific effects of the live MMR vaccine were masked by the simultaneous co-administration of the attenuated MenC vaccine. The Danish study that reported non-specific effects of MMR vaccination studied only MMR vaccination and not the combination of MMR and MenC, which could also explain the different findings with our study. Other European countries might also have the opportunity to study non-specific effects of vaccination and the potential for healthy vaccinee bias. As in the Netherlands, many European countries first give a vaccine containing DTP, mostly around age 12 months, and thereafter a MMR vaccine at age 15-18 months (ECDC: http://vaccineschedule.ecdc.europa.eu/). There are also some countries, however, that first give the MMR vaccine and then the DTP vaccine, although in most of these countries the MMR vaccine is given together with PCV or MenC vaccination. Nevertheless, it would be interesting to see the results of a similar analysis from countries with a "reversed" schedule.

\section{Strengths and weaknesses of study}

A major strength of our study is that it is nationwide population based and included more than a million children. Because nearly all children received both the DTaP-IPV-Hib+PCV and MMR+MenC vaccinations, children acted as their own controls. Analyses were stratified by date of birth to fully control for age, year, and season. The large sample size enabled us to perform multiple stratified analyses. Moreover, the Dutch vaccination system, which is characterised by its fixed schedule as a result of prescheduled appointments, provided the opportunity to explore and illustrate the presence of healthy vaccinee bias. A limitation of the study is that we did not have information on the reason for delayed vaccination. Moreover, it should be noted that not all hospital admissions were captured in the national medication registration and that completeness decreased with time (97\% in 2005 to $75 \%$ in 2012). ${ }^{44}$ We assumed that the completeness was not associated with the timing of vaccination in our study and therefore would not confound the results. The interaction test confirmed this; we observed no effect modification for birth cohort. As these findings were based on admission data, we took only severely affected children into account. Therefore, it would be of interest to investigate the association between most recent vaccination and GP consultations for infections. 
As this would enable us to take into account acute illness (for example, a GP visit for fever, as vaccination should be avoided if a child's temperature is $\geq 38.5^{\circ} \mathrm{C}^{50}$ ) as a time varying variable, it could provide more insight in the effect of acute illness as part of healthy vaccinee bias.

In conclusion, throughout the world vaccination has contributed to a decline in mortality and morbidity as result of specific vaccine effects. Evidence for nonspecific effects, however, particularly in high income countries, remains uncertain. In our observational study on non-specific effects of vaccination in more than a million Dutch children, a lower rate of hospital admission for infection followed receipt of an additional vaccination (and therefore adherence to the routinely recommended schedule) and could not be attributed to MMR specifically. Receipt of MMR+MenC as most recent vaccination was associated with a $38 \%$ lower rate of admission related to infectious disease, compared with DTaP-IPV-Hib+PCV as most recent vaccination. We also observed a 31\% lower rate of admission for infection with receipt of the fourth DTaPIPV-Hib+PCV as most recent vaccination, compared with the third as most recent vaccination. These findings, together with those for the negative control outcome, suggest that the delay of a vaccination (be it DTaP-IPV-Hib+PCV or MMR+MenC) might depend on the health status of a child, rather than the other way around. We cannot exclude the presence of non-specific effects of MMR vaccination, but we are unable to disentangle the possible non-specific effects from bias. Our findings emphasise the importance of interpreting findings from observational studies on non-specific effects of vaccination with great caution.

Contributors: SMAJT, HEdM, SJMH, FRMvdK, EAMS, MABvdS, and MJK contributed to the conception and design of the study. SMAIT and MIK acquired and interpreted the data. SMAJT and AGCB carried out the statistical analysis. SMAIT drafted the manuscript, which was critically revised for important intellectual content by all authors, who approved the final version. SMAJT and MJK are guarantors.

Funding: This research was funded by the Dutch Ministry of Health, and was carried out in the framework of RIVM Strategic Programme (SPR), in which expertise and innovative projects prepare RIVM to respond to future issues in health and sustainability. The views expressed in this publication are those of the authors and not necessarily those of the funding agencies.

Competing interests: All authors have completed the ICMJE uniform disclosure form at www.icmje.org/coi_disclosure.pdf and declare: support from the Dutch Ministry of Health for the submitted work: no financial relationships with any organisations that might have an interest in the submitted work in the previous three years; no other relationships or activities that could appear to have influenced the submitted work.

Ethical approval: Not required

Transparency: The corresponding author (MK) affirms that the manuscript is an honest, accurate, and transparent account of the study being reported, no important aspects of the study have been omitted, and any discrepancies from the study as planned have been explained.

Data sharing: The statistical code is available in appendix 2 Individual data are not available because of privacy reasons.

This is an Open Access article distributed in accordance with the Creative Commons Attribution Non Commercial (CC BY-NC 4.0) license, which permits others to distribute, remix, adapt, build upon this work non-commercially, and license their derivative works on different terms, provided the original work is properly cited and the use is noncommercial. See: http://creativecommons.org/licenses/by-nc/4.0/.
1 van Wijhe M, McDonald SA, de Melker HE, Postma MJ, Wallinga J. Effect of vaccination programmes on mortality burden among children and young adults in the Netherlands during the 20th century: a historical analysis. Lancet Infect Dis 2016;16:592-8. doi:10.1016/S1473-3099(16)00027-X

2 Roush SW, Murphy TVVaccine-Preventable Disease Table Working Group. Historical comparisons of morbidity and mortality for vaccinepreventable diseases in the United States. JAMA 2007:298:2155-63. doi:10.1001/jama.298.18.2155

3 Flanagan KL, van Crevel R, Curtis N, Shann F, Levy OOptimmunize Network. Heterologous ("nonspecific") and sex-differential effects of vaccines: epidemiology, clinical trials, and emerging immunologic mechanisms. Clin Infect Dis 2013:57:283-9. doi:10.1093/cid/ cit209

4 Kristensen I, Aaby P, Jensen H. Routine vaccinations and child survival: follow up study in Guinea-Bissau, West Africa. BMI 2000.321:1435-8 doi:10.1136/bmj.321.7274.1435

5 Aaby P, Samb B, Simondon F, Seck AM, Knudsen K, Whittle H. Non-specific beneficial effect of measles immunisation: analysis of mortality studies from developing countries. BMJ 1995;311:481-5. doi:10.1136/bmi.311.7003.481

6 Aaby P, Martins CL, Garly ML. Non-specific effects of standard measles vaccine at 4.5 and 9 months of age on childhood mortality: randomised controlled trial. BMJ 2010;341:c6495. doi:10.1136/ bmj.c6495

7 Aaby P, Benn C, Nielsen J, Lisse IM, Rodrigues A, Ravn H. Testing the hypothesis that diphtheria-tetanus-pertussis vaccine has negative non-specific and sex-differential effects on child survival in highmortality countries. BMJ Open 2012;2:e000707. doi:10.1136/ bmjopen-2011-000707

8 Shann F, Nohynek H, Scott JA, Hesseling A, Flanagan KLWorking Group on Nonspecific Effects of Vaccines. Randomized trials to study the nonspecific effects of vaccines in children in low-income countries. Pediatr Infect Dis / 2010;29:457-61. doi:10.1097/ INF.0b013e3181c91361

9 Shann F. The non-specific effects of vaccines. Arch Dis Child 2010;95:662-7. doi:10.1136/adc.2009.157537

10 Sørup S, Benn CS, Poulsen A, Krause TG, Aaby P, Ravn H. Live vaccine against measles, mumps, and rubella and the risk of hospital admissions for nontargeted infections. JAMA 2014;311:826-35. doi:10.1001/jama.2014.470

11 Aaby P, Nielsen J, Benn CS, Trape JF. Sex-differential and non-specific effects of routine vaccinations in a rural area with low vaccination coverage: an observational study from Senegal. Trans R Soc Trop Med Hyg 2015;109:77-84. doi:10.1093/trstmh/tru186

12 Aaby P, Vessari H, Nielsen J. Sex differential effects of routine immunizations and childhood survival in rural Malawi. Pediatr Infect Dis / 2006;25:721-7. doi:10.1097/01.inf.0000227829.64686.ae

13 Hirve S, Bavdekar A, Juvekar S, Benn CS, Nielsen J, Aaby P. Nonspecific and sex-differential effects of vaccinations on child survival in rural western India. Vaccine 2012;30:7300-8. doi:10.1016/j. vaccine.2012.09.035

14 Fisker AB, Ravn H, Rodrigues A. Co-administration of live measles and yellow fever vaccines and inactivated pentavalent vaccines is associated with increased mortality compared with measles and yellow fever vaccines only. An observational study from Guinea-Bissau. Vaccine 2014;32:598-605. doi:10.1016/j. vaccine.2013.11.074

15 Aaby P, Biai S, Veirum JE. DTP with or after measles vaccination is associated with increased in-hospital mortality in Guinea-Bissau. Vaccine 2007;25:1265-9. doi:10.1016/j.vaccine.2006.10.007

16 Sørup S, Benn CS, Poulsen A, Krause TG, Aaby P, Ravn H. Simultaneous vaccination with MMR and DTaP-IPV-Hib and rate of hospital admissions with any infections: A nationwide registe based cohort study. Vaccine 2016;34:6172-80. doi:10.1016/j. vaccine.2016.11.005

17 Blok BA, Arts RJ, van Crevel R, Benn CS, Netea MG. Trained innate immunity as underlying mechanism for the long-term, nonspecific effects of vaccines. / Leukoc Biol 2015:98:347-56 doi:10.1189/ jlb.5RI0315-096R

18 Goodridge HS, Ahmed SS, Curtis N. Harnessing the beneficial heterologous effects of vaccination. Nat Rev Immunol 2016;16: 392-400. doi:10.1038/nri.2016.43

19 Sørup S, Benn CS, Stensballe LG, Aaby P, Ravn H. Measles-mumpsrubella vaccination and respiratory syncytial virus-associated hospital contact. Vaccine 2015;33:237-45. doi:10.1016/j. vaccine.2014.07.110

20 Farrington CP, Firth MJ, Moulton LH, Ravn H, Andersen PK, Evans SWorking Group on Non-specific Effects of Vaccines. Epidemiological studies of the non-specific effects of vaccines: II--methodological issues in the design and analysis of cohort studies. Trop Med Int Health 2009:14:977-85 doi:10.1111/j.1365-3156.2009.02302x

21 Higgins JP, Soares-Weiser K, López-López JA. Association of BCG, DTP, and measles containing vaccines with childhood mortality: systematic review. BMJ 2016;355:i5170. doi:10.1136/bmj.i5170 
22 Lehmann D, Vail J, Firth MJ, de Klerk NH, Alpers MP. Benefits of routine immunizations on childhood survival in Tari, Southern Highlands Province, Papua New Guinea. Int J Epidemiol 2005;34:138-48. doi:10.1093/ije/dyh262

23 Moulton LH, Rahmathullah L, Halsey NA, Thulasiraj RD, Katz J, Tielsch JM. Evaluation of non-specific effects of infant immunizations on early infant mortality in a southern Indian population. Trop Med Int Health 2005:10:947-55 doi:10.1111/i.1365-3156.2005.01434.

24 Aaby P, Ravn H, Roth A. Early diphtheria-tetanus-pertussis vaccination associated with higher female mortality and no difference in male mortality in a cohort of low birthweight children: an observational study within a randomised trial. Arch Dis Child 2012;97:685-91. doi:10.1136/archdischild-2011-300646

25 Aaby P, Jensen H, Garly ML, Balé C, Martins C, Lisse I. Routine vaccinations and child survival in a war situation with high mortality: effect of gender. Vaccine 2002;21:15-20. doi:10.1016/S0264410X(02)00441-3

26 Aaby P, Jensen H, Gomes J, Fernandes M, Lisse IM. The introduction of diphtheria-tetanus-pertussis vaccine and child mortality in rural Guinea-Bissau: an observational study. Int J Epidemiol 2004;33: 374-80. doi:10.1093/ije/dyh005

27 Vaugelade J, Pinchinat S, Guiella G, Elguero E, Simondon F. Non-specific effects of vaccination on child survival: prospective cohort study in Burkina Faso. BMJ 2004;329:1309. doi:10.1136/ bmi.38261.496366.82

28 Velema JP, Alihonou EM, Gandaho T, Hounye FH. Childhood mortality among users and non-users of primary health care in a rural west African community. Int J Epidemiol 1991;20:474-9. doi:10.1093/ ije/20.2.474

29 Hartfield J, Morley D. Efficacy of measles vaccine. J Hyg (Lond) 1963;61:143-7. doi:10.1017/S0022172400020817

30 Benn CS, Balé C, Sommerfelt H, Friis H, Aaby P. Hypothesis: Vitamin A supplementation and childhood mortality: amplification of the non-specific effects of vaccines?/nt J Epidemiol 2003;32:822-8. doi:10.1093/ije/dyg208

31 Aaby P, Garly ML, Nielsen J. Increased female-male mortality ratio associated with inactivated polio and diphtheria-tetanus-pertussis vaccines: Observations from vaccination trials in GuineaBissau. Pediatr Infect Dis / 2007;26:247-52. doi:10.1097/01 inf.0000256735.05098.01

32 Van Balen H, Mercenier P, Daveloose PThe Kasongo Project Team. Influence of measles vaccination on survival pattern of 7--35-monthold children in Kasongo, Zaire. Lancet 1981;1:764-7.

33 Aaby P, Samb B, Simondon F. Divergent mortality for male and female recipients of low-titer and high-titer measles vaccines in rural Senegal. Am J Epidemiol 1993;138:746-55. doi:10.1093/ oxfordjournals.aje.a116912

34 Kabir Z, Long J, Reddaiah VP, Kevany J, Kapoor SK. Non-specific effect of measles vaccination on overall child mortality in an area of rural India with high vaccination coverage: a population-based casecontrol study. Bull World Health Organ 2003;81:244-50.

35 George K, Joseph A, Muliyil J, Abraham S, Bhattacharji S, John KR. Measles vaccination before nine months. Trop Med Int Health 1998;3:751-6. doi:10.1046/j.1365-3156.1998.00295.x

36 Holt EA, Boulos R, Halsey NA, Boulos LM, Boulos C. Childhood survival in Haiti: protective effect of measles vaccination. Pediatrics 1990;85:188-94
37 Fisker AB, Hornshøj L, Rodrigues A. Effects of the introduction of new vaccines in Guinea-Bissau on vaccine coverage, vaccine timeliness, and child survival: an observational study. Lancet Glob Health 2014;2:e478-87. doi:10.1016/S2214 109X(14)70274-8

38 Aaby P, Knudsen K, Jensen TG. Measles incidence, vaccine efficacy, and mortality in two urban African areas with high vaccination coverage. / Infect Dis 1990;162:1043-8. doi:10.1093/ infdis/162.5.1043

39 Aaby P, Bukh J, Lisse IM, Smits AJ. Measles vaccination and reduction in child mortality: a community study from Guinea-Bissau. I Infect 1984;8:13-21. doi:10.1016/S0163-4453(84)93192-X

40 Aaby P, Bukh J, Lisse IM. Determinants of measles mortality in a rural area of Guinea-Bissau: crowding, age, and malnutrition. J Trop Pediatr 1984;30:164-8. doi:10.1093/tropej/30.3.164

41 Aaby P, Bhuiya A, Nahar L, Knudsen K, de Francisco A, Strong M. The survival benefit of measles immunization may not be explained entirely by the prevention of measles disease: a community study from rural Bangladesh. Int J Epidemiol 2003;32:106-16. doi:10.1093/ije/dyg005

42 Breiman RF, Streatfield PK, Phelan M, Shifa N, Rashid M, Yunus M. Effect of infant immunisation on childhood mortality in rura Bangladesh: analysis of health and demographic surveillance data. Lancet 2004;364:2204-11. doi:10.1016/S0140-6736(04) 17593-4

43 van Lier A, Oomen P, de Hoogh P. Præventis, the immunisation register of the Netherlands: a tool to evaluate the National Immunisation Programme. Euro Surveill 2012;17:20153.

44 Documentatierapporten LMR (Documentation reports Nationa Medical Register). Centraal Bureau voor de Statistiek (Statistics Netherlands), 2005-12

45 Bruin de A, Ariel A, Verweij G, Israëls A. Methode van bijschatten van StatLinetabel Ziekenhuispatiënten naar diagnose. Den Haag/Heerlen: Centraal Bureau voor de Statistiek, 2009.

46 Lipsitch M, Tchetgen Tchetgen E, Cohen T. Negative controls: a tool for detecting confounding and bias in observational studies. Epidemiology 2010;21:383-8. doi:10.1097/ EDE.0b013e3181d61eeb

47 Andersen PK, Gill RD. Cox's Regression Model for Counting Processes: A Large Sample Study. Ann Stat 1982;10:1100-20doi:10.1214 aos/1176345976.

48 Stensballe LG, Sørup S, Aaby P. BCG vaccination at birth and early childhood hospitalisation: a randomised clinical multicentre trial. Arch Dis Child 2017;102:224-31. doi:10.1136/ archdischild-2016-310760

49 Kjærgaard J, Birk NM, Nissen TN. Nonspecific effect of BCG vaccination at birth on early childhood infections: a randomized, clinical multicenter trial. Pediatr Res 2016:80:681-5. doi:10.1038/ pr.2016.142

50 National Institute for Public Health and the Environment (RIVM). [Guidelines for contraindications for vaccination]. http://www.rivm.nl/ Documenten en_publicaties/Professioneel_Praktisch/Richtlijnen/ Infectieziekten/Rijksvaccinatieprogramma/Uitvoeringsregels RVP_2015_2016/Inhoud/2_Contra_indicaties.

Appendix 1: Supplementary tables Appendix 2: Supplementary information 